\title{
Defective Edge states and Anomalous Bulk-boundary Correspondence for Topological Insulators under Non-Hermitian Similarity Transformation
}

\author{
Can Wang, ${ }^{1}$ Xiao-Ran Wang, ${ }^{1}$ Cui-Xian Guo, ${ }^{1}$ and Su-Peng Kou, ${ }^{1}$ * \\ ${ }^{1}$ Center for Advanced Quantum Studies, Department of Physics, \\ Beijing Normal University, Beijing 100875, China
}

\begin{abstract}
It was known that for non-Hermitian topological systems due to the non-Hermitian skin effect, the bulk-edge correspondence is broken down. In this paper, by using one-dimensional Su-SchriefferHeeger model and two-dimensional (deformed) Qi-Wu-Zhang model as examples, we focus on a special type of non-Hermitian topological system without non-Hermitian skin effect - topological systems under non-Hermitian similarity transformation. In these non-Hermitian systems, the defective edge states and the breakdown of bulk-edge correspondence are discovered. To characterize the topological properties, we introduce a new type of inversion symmetry-protected topological invariant - total $Z_{2}$ topological invariant. In topological phases, defective edge states appear. With the help of the effective edge Hamiltonian, we find that the defective edge states are protected by (generalized) chiral symmetry and thus the (singular) defective edge states are unstable against the perturbation breaking the chiral symmetry. In addition, the results are generalized to nonHermitian topological insulators with inversion symmetry in higher dimensions. This work could help people to understand the defective edge states and the breakdown of bulk-edge correspondence for non-Hermitian topological systems.
\end{abstract}

\section{INTRODUCTION}

Non-Hermitian topological systems 1 60 have been confirmed with diverse peculiar underlying physics distinguishing from its Hermitian counterparts 61 65]. Generally, the non-Hermitian terms i.e., imaginary-mass, or imaginary-momentum, or anti-commutating in tightbinding topological model bring novel properties such as complex spectra, defective edge states [9, 23], nonHermitian skin effect 20, 32, 39, 54, 56], ... Due to the conventional bulk-boundary correspondence (BBC) collapsed in non-Hermitian topological insulators 17, 20 22, 47 49, 53, 56], the non-Bloch topological invariants [20. and the effective theory for the edge states $[60$. were introduced to describe the non-Hermitian topological systems. Within the framework of Altland-Zirnbauer (AZ) theory, the topological invariants classifications based on different symmetries in the non-Hermitian topological systems were developed [19, 43, 44]. After considering reflection symmetry, the classification of non-Hermitian topological systems is also finished 45 ].

However, a large class of non-Hermitian topological systems with real energy spectra have not been discussed: topological system under non-Hermitian similarity transformation. In particular, under non-Hermitian similarity transformation, the energy levels are all real and same to those of the Hermitian counterparts 66, 67. . So, there doesn't exist the non-Hermitian skin effect. However, the quantum states of these systems may be quite different from that of the Hermitian counterpart. Here, to completely understand the underlying physics of this type of non-Hermitian topological systems, we ask the following questions:

\footnotetext{
${ }^{*}$ Corresponding author; Electronic address: spkou@bnu.edu.cn
}

1. What are the topological invariants in the bulk for this type of non-Hermitian topological systems?

2. Does there exist the defective edge states in this type of non-Hermitian topological systems without non-Hermitian skin effect?

3. How to accurately characterize the physics of edge states and the possible anomalous BBC of defective edge states?

4. Are the defective edge states in this non-Hermitian topological system stable?

In this paper, to answer above questions, we systematically study the non-Hermitian topological systems by investigating one-dimensional (1D) Su-Schrieffer-Heeger (SSH) model and two-dimensional (2D) Qi-Wu-Zhang (QWZ) model under non-Hermitian similarity transformation. To characterize these non-Hermitian topological systems, we introduce a new type of symmetry-protected topological invariant - total $Z_{2}$ topological invariant. In topological phases, defective edge states appear, i.e., edge states on the ends of finite non-Hermitian topological systems with non-Hermitian coalescence. To accurately characterize the physics of defective edge states and the anomalous BBC, the effective edge Hamiltonians are obtained. With the help of the effective edge Hamiltonian, we find that the defective edge states are protected by (generalized) chiral symmetry and the (singular) defective edge states are unstable against the perturbation breaking the chiral symmetry. In addition, we generalize the results to non-Hermitian topological insulators in higher dimensions.

This paper is organized as follows. In Sec. II, we explore the defective edge states and the anomalous BBC for $1 \mathrm{D} \mathrm{Su-Schrieffer-Heeger} \mathrm{under} \mathrm{non-Hermitian} \mathrm{simi-}$ larity transformation. In Sec. III, we study the defective 
edge states and the anomalous BBC for 2D Chern insulator under non-Hermitian similarity transformation. In Sec. IV we generalize the theory to topological insulators in higher dimensions under non-Hermitian similarity transformation. In the end, we give a brief conclusion in Sec. V.

\section{ANOMALOUS BBC AND DEFECTIVE EDGE STATES FOR 1D SU-SCHRIEFFER-HEEGER UNDER NON-HERMITIAN SIMILARITY TRANSFORMATION}

\section{A. 1D Hermitian Su-Schrieffer-Heeger model}

We begin with the simplest 1D Topological insulator - Hermitian SSH model, of which tight-binding Hamiltonian for the finite system with $N$ pairs of lattice sites is given by

$H_{0}^{\mathrm{SSH}}=t_{1} \sum_{n=1}^{N}|n, B\rangle\left\langle n, A\left|+t_{2} \sum_{n=1}^{N-1}\right| n+1, A\right\rangle\langle n, B|+$ h.c.

where $A(B)$ represents the sublattices and $n$ indicates the $n$-th cell of the lattice. Correspondingly, under periodic boundary condition, its Bloch Hamiltonian becomes

$$
\begin{aligned}
H_{0}^{\mathrm{SSH}}(k) & =\left(t_{1}+t_{2} \cos k\right) \sigma_{x}+\left(t_{2} \sin k\right) \sigma_{y} \\
& =\left(\begin{array}{cc}
0 & t_{1}+t_{2} e^{-i k} \\
t_{1}+t_{2} e^{i k} & 0
\end{array}\right),
\end{aligned}
$$

where the real parameters $t_{1}, t_{2}$ are the intra (inter)hopping amplitude, $\sigma_{i}$ refers to the Pauli matrices and the $2 \times 2$ matrix above is defined as the bulk Hamiltonian $H_{0}^{\mathrm{SSH}}(k)$. Under periodic boundary condition, the energy spectra are

$$
E(k)= \pm \sqrt{\left(t_{1}+t_{2} \cos k\right)^{2}+\left(t_{2} \sin k\right)^{2}} .
$$

Fig. 1(b) shows the energy spectra of SSH model.

\section{Chiral symmetry and inversion symmetry} i.e.

The Hamiltonian $H_{0}^{\mathrm{SSH}}(k)$ possesses chiral symmetry,

$$
\sigma_{z} H_{0}^{\mathrm{SSH}}(k) \sigma_{z}=-H_{0}^{\mathrm{SSH}}(k) .
$$

$H_{0}^{\mathrm{SSH}}(k)$ also has inversion symmetry satisfying the relation

$$
\hat{\mathcal{I}} H_{0}^{\mathrm{SSH}}(k) \hat{\mathcal{I}}^{-1}=H_{0}^{\mathrm{SSH}}(-k)
$$

where the inversion operator $\hat{\mathcal{I}}$ is $\sigma_{x}$. This equation implies

$$
E(k)=E(-k)
$$

\section{Total $Z_{2}$ topological invariant}

According to AZ classification, it is 1D AIII type, of which the topological invariant is a winding number

$$
w=\frac{1}{2 \pi} \int_{-\pi}^{\pi} \partial_{k} \phi_{n}(k) \cdot d k .
$$

where $\phi_{n}(k)=\tan ^{-1}\left(d_{y} / d_{x}\right)$ with $d_{y}=t_{2} \sin k$ and $d_{x}=$ $t_{1}+t_{2} \cos k$. There are two phases, topological phase with $w=1$ in the region of $\left|t_{1}\right|<\left|t_{2}\right|$ and trivial phase with $w=0$ in the region of $\left|t_{1}\right|>\left|t_{2}\right|$. At $\left|t_{1}\right|=\left|t_{2}\right|$, there exists a topological phase transition. See the phase diagram in Fig. 1(a) $(\beta=0$ case).

However, in this paper, to characterize the SSH model with chiral symmetry and inversion symmetry, instead of winding number $w$, we introduce a new topological invariant - total $Z_{2}$ topological invariant $\eta=\eta_{k=0} \eta_{k=\pi}$. See the detailed definition in below discussion.

Now, the Bloch Hamiltonian is divided into three parts

$$
\begin{aligned}
H_{0}^{\mathrm{SSH}}(k) & =H_{0}^{\mathrm{SSH}}(k \neq 0 / \pi)+H_{0}^{\mathrm{SSH}}(k=0) \\
+ & H_{0}^{\mathrm{SSH}}(k=\pi)
\end{aligned}
$$

Here, $k=0 / \pi$ are the high symmetry points in momentum space. The quantum states at these high symmetry points are invariant under inversion operation, i.e.,

$$
\begin{aligned}
& \hat{\mathcal{I}}|\psi(k=0)\rangle=|\psi(k=0)\rangle, \\
& \hat{\mathcal{I}}|\psi(k=\pi)\rangle=|\psi(k=\pi)\rangle .
\end{aligned}
$$

For $k=0$, we have

$$
\frac{1}{2} \operatorname{Tr}\left[\hat{\mathcal{I}} \cdot H_{0}^{\mathrm{SSH}}(k=0)\right]=t_{1}+t_{2}
$$

for $k=\pi$, we have

$$
\frac{1}{2} \operatorname{Tr}\left[\hat{\mathcal{I}} \cdot H_{0}^{\mathrm{SSH}}(k=\pi)\right]=t_{1}-t_{2}
$$

To describe the topological structure of $H_{0}^{\mathrm{SSH}}(k)$, we define two $Z_{2}$ topological invariants,

$$
\eta_{k=0}=\frac{\operatorname{Tr}\left[\hat{\mathcal{I}} \cdot H_{0}^{\mathrm{SSH}}[k=0]\right]}{\left|\operatorname{Tr}\left[\hat{\mathcal{I}} \cdot H_{0}^{\mathrm{SSH}}[k=0]\right]\right|}=\frac{t_{1}+t_{2}}{\left|t_{1}+t_{2}\right|}
$$

and

$$
\eta_{k=\pi}=\frac{\operatorname{Tr}\left[\hat{\mathcal{I}} \cdot H_{0}^{\mathrm{SSH}}[k=\pi]\right]}{\left|\operatorname{Tr}\left[\hat{\mathcal{I}} \cdot H_{0}^{\mathrm{SSH}}[k=\pi]\right]\right|}=\frac{t_{1}-t_{2}}{\left|t_{1}-t_{2}\right|}
$$

where $\hat{\mathcal{I}}=\sigma_{x}$. Now, we use the number ' 1 ' to denote the case $\eta_{k=0}=-1$ or $\eta_{k=\pi}=-1$ and the number ' 0 ' to denote the case $\eta_{k=0}=1$ or $\eta_{k=\pi}=1$. Hence, there are 4 different universal classes of topological phases denoted by (11), (00), (10), (01) [68]. The following table shows 

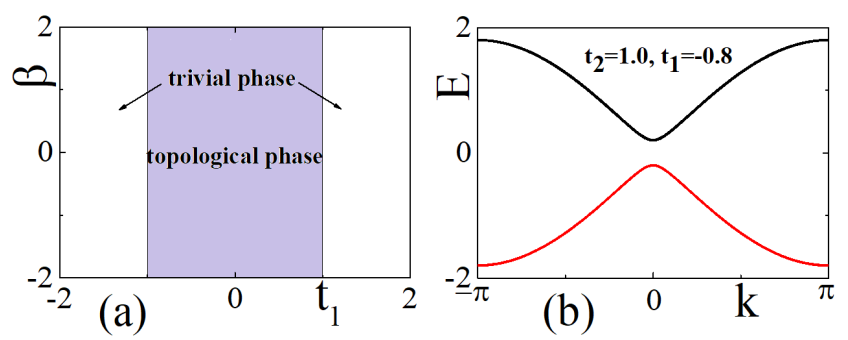

FIG. 1: (Color online) (a) Global phase diagram for 1D SSH model under non-Hermitian similarity transformation. The purple area represents the topological phase with $\eta=-1$, while the white space represents the trivial phase with $\eta=$ 1 , respectively. The topological phase transition occurs at $t_{1}=t_{2}$ (b) The energy spectra for 1D SSH model under nonHermitian similarity transformation. The energy spectra are independent on $\beta$.

the $Z_{2}$ topological invariants of different universal classes of topological phases

$$
\begin{array}{lllll} 
& (11) & (10) & (01) & (00) \\
k=0 & -1 & -1 & 1 & 1 \\
k=\pi & -1 & 1 & -1 & 1
\end{array}
$$

The total $Z_{2}$ topological invariant is defined as

$$
\eta=\eta_{k=0} \eta_{k=\pi}=\left\{\begin{array}{l}
+1, \text { trivial phase } \\
-1, \text { topological phase }
\end{array} .\right.
$$

$\eta$ becomes a topological invariant to characterize the universal properties of different topological phases for SSH model with inversion symmetry. There are 2 trivial phases: (11), (00), and 2 topological phases: (10), (01). In particular, the phase diagram from the $Z_{2}$ topological invariant $\eta$ and that from the winding number $\omega$ are same, i.e., topological phases in the region of $\left|t_{1}\right|<\left|t_{2}\right|$ and trivial phases in the region of $\left|t_{1}\right|>\left|t_{2}\right|$.

\section{Effective edge Hamiltonian}

The non-zero winding number or total $Z_{2}$ topological invariant $\eta$ guarantees the edge states with zero energy for a system in thermodynamical limit, $N \rightarrow \infty$. For the case of non-topological phase, the edge states disappear. This leads to the conventional bulk-boundary correspondence for Hermitian topological systems.

In topological phase, we consider the edge states $\left|\mathrm{e}_{0}^{\mathrm{L}}\right\rangle$ and $\left|\mathrm{e}_{0}^{\mathrm{R}}\right\rangle$ on the left and right ends of the semi-infinite chain as the basis [69], i.e.,

$$
\left|\mathrm{e}_{0}^{\mathrm{L}}\right\rangle=\frac{1}{\mathcal{N}} \sum_{n=1}^{N-1}\left(\frac{t_{1}}{t_{2}}\right)^{n-1}|n\rangle \otimes(1,0)^{\mathrm{T}}
$$

and

$$
\left|\mathrm{e}_{0}^{\mathrm{R}}\right\rangle=\frac{1}{\mathcal{N}} \sum_{n=0}^{N-1}\left(\frac{t_{1}}{t_{2}}\right)^{n}|N-n\rangle \otimes(0,1)^{\mathrm{T}},
$$

where the normalization factor is

$$
\mathcal{N}=\sqrt{\left(1-\left(\frac{t_{1}}{t_{2}}\right)^{2 N}\right) /\left(1-\left(\frac{t_{1}}{t_{2}}\right)^{2}\right)} .
$$

$(1,0)^{\mathrm{T}}$ and $(0,1)^{\mathrm{T}}$ denote the state vectors of twosublattices. For an semi-infinite chain, we have $N \rightarrow \infty$. In general, the wave-function for an edge state can be written as a superposition of the two end states at the left and right ends

$$
\left|\psi_{0}\right\rangle=C_{1}\left|\mathrm{e}_{0}^{\mathrm{L}}\right\rangle+C_{2}\left|\mathrm{e}_{0}^{\mathrm{R}}\right\rangle
$$

where $C_{1}$ and $C_{2}$ are complex numbers, and $\left|C_{1}\right|^{2}+$ $\left|C_{2}\right|^{2}=1$.

To characterize the two edge states, we introduce an effective edge Hamiltonian,

$$
\hat{\mathcal{H}}_{\mathrm{eff}}=\left(\begin{array}{cc}
\varepsilon_{L L} & \varepsilon_{L R} \\
\varepsilon_{R L} & \varepsilon_{R R}
\end{array}\right)
$$

where

$$
\begin{aligned}
& \varepsilon_{L L}=\left\langle\mathrm{e}_{0}^{\mathrm{L}}\left|H_{0}^{\mathrm{SSH}}\right| \mathrm{e}_{0}^{\mathrm{L}}\right\rangle, \varepsilon_{L R}=\left\langle\mathrm{e}_{0}^{\mathrm{L}}\left|H_{0}^{\mathrm{SSH}}\right| \mathrm{e}_{0}^{\mathrm{R}}\right\rangle, \\
& \varepsilon_{R L}=\left\langle\mathrm{e}_{0}^{\mathrm{R}}\left|H_{0}^{\mathrm{SSH}}\right| \mathrm{e}_{0}^{\mathrm{L}}\right\rangle, \varepsilon_{R R}=\left\langle\mathrm{e}_{0}^{\mathrm{R}}\left|H_{0}^{\mathrm{SSH}}\right| \mathrm{e}_{0}^{\mathrm{R}}\right\rangle .
\end{aligned}
$$

For this model, we have

$$
\begin{aligned}
& \varepsilon_{L L}=-\varepsilon_{R R}=0, \\
& \varepsilon_{L R}=\varepsilon_{R L}=\Delta=\frac{\left(t_{2}^{2}-t_{1}^{2}\right)}{t_{2}}\left(\frac{t_{1}}{t_{2}}\right)^{N} .
\end{aligned}
$$

The effective edge Hamiltonian becomes

$$
\hat{\mathcal{H}}_{\mathrm{eff}}=\Delta \cdot \tau^{x}
$$

where $\tau^{i}$ is the Pauli matrix acting on the two edge states. As a result, the energy levels for the two edge states are

$$
E_{ \pm}= \pm \frac{\left(t_{2}^{2}-t_{1}^{2}\right)}{t_{2}}\left(\frac{t_{1}}{t_{2}}\right)^{N}
$$

and their eigenstates are

$$
\frac{1}{\sqrt{2}}\left(\left|\mathrm{e}_{0}^{\mathrm{L}}\right\rangle+\left|\mathrm{e}_{0}^{\mathrm{R}}\right\rangle\right), \frac{1}{\sqrt{2}}\left(\left|\mathrm{e}_{0}^{\mathrm{L}}\right\rangle-\left|\mathrm{e}_{0}^{\mathrm{R}}\right\rangle\right) .
$$

\section{B. 1D SSH model under non-Hermitian similarity transformation}

In the second step, we consider the SSH model under the non-Hermitian similarity transformation,

$$
S=\left(\begin{array}{cc}
1 & 0 \\
0 & e^{\beta}
\end{array}\right)
$$


where $\beta$ denotes the non-Hermiticity strength. Under the non-Hermitian similarity transformation, we have

$$
\begin{aligned}
\sigma_{x} & =\left(\begin{array}{ll}
0 & 1 \\
1 & 0
\end{array}\right) \rightarrow\left(\sigma_{x}\right)^{\beta}=S^{-1} \sigma_{x} S \\
& =\cosh (\beta) \sigma_{x}+i \sinh (\beta) \sigma_{y}=\left(\begin{array}{cc}
0 & e^{\beta} \\
e^{-\beta} & 0
\end{array}\right), \\
\sigma_{y}= & \left(\begin{array}{cc}
0 & -i \\
i & 0
\end{array}\right) \rightarrow\left(\sigma_{y}\right)^{\beta}=S^{-1} \sigma_{y} S \\
& =\cosh (\beta) \sigma_{y}-i \sinh (\beta) \sigma_{x}=\left(\begin{array}{cc}
0 & -i e^{\beta} \\
i e^{-\beta} & 0
\end{array}\right),
\end{aligned}
$$

and

$$
\sigma_{z} \rightarrow\left(\sigma_{z}\right)^{\beta}=S^{-1} \sigma_{z} S=\sigma_{z}
$$

As a result, under the non-Hermitian similarity transformation, the Bloch Hamiltonian of SSH model turns into a nonreciprocal one, that is

$$
\begin{aligned}
H_{0}^{\mathrm{SSH}} & \rightarrow H^{\mathrm{SSH}}=S^{-1} H_{0}^{\mathrm{SSH}} S \\
& =\left(\begin{array}{cc}
0 & e^{\beta}\left(t_{1}+t_{2} e^{-i k}\right) \\
e^{-\beta}\left(t_{1}+t_{2} e^{i k}\right) & 0
\end{array}\right) \\
& =\left(t_{1} \cosh \beta+t_{2} \cos (k+i \beta)\right) \sigma_{x} \\
& +\left(i t_{1} \sinh \beta+t_{2} \sin (k+i \beta)\right) \sigma_{y}
\end{aligned}
$$

To describe the edge states, we rewrite the Hamiltonian in real space

$$
\begin{aligned}
H^{\mathrm{SSH}} & =\sum_{n} t_{1 L}|n, A\rangle\left\langle n, B\left|+t_{2 R}\right| n, A\right\rangle\langle n-1, B| \\
& +t_{1 R}|n, B\rangle\left\langle n, A\left|+t_{2 L}\right| n, B\right\rangle\langle n+1, A|,
\end{aligned}
$$

where $t_{1(2) L(R)}$ is the right (left) intra (inter)-hopping amplitude. Then, the effective hopping parameters in real space become

$$
\begin{aligned}
& t_{1 L}=t_{1} e^{\beta}, t_{1 R}=t_{1} e^{-\beta} \\
& t_{2 L}=t_{2} e^{-\beta}, t_{2 R}=t_{2} e^{\beta}
\end{aligned}
$$

The eigenvalues of the Hamiltonian $H^{\mathrm{SSH}}$ for the nonreciprocal $\mathrm{SSH}$ model are same to those of the Hermitian $\mathrm{SSH}$ model, i.e.,

$$
E_{ \pm}(k)= \pm \sqrt{\left(t_{1}+t_{2} \cos k\right)^{2}+\left(t_{2} \sin k\right)^{2}}
$$

Fig. 1(b) also shows the energy spectra of SSH model under non-Hermitian similarity transformation that are all real and unchanged with $\beta$. As a result, due to the real energy spectra, there doesn't exist the non-Hermitian skin effect.

\section{Total $Z_{2}$ topological invariant}

For SSH model under non-Hermitian similarity transformation, the Hamiltonian also has chiral symmetry, i.e., $\sigma_{z} H^{\mathrm{SSH}}(k) \sigma_{z}=-H^{\mathrm{SSH}}(k)$ and inversion symmetry, i.e., $\hat{\mathcal{I}} H^{\mathrm{SSH}}(k) \hat{\mathcal{I}}^{-1}=H^{\mathrm{SSH}}(-k)$ where the inversion operator $\hat{\mathcal{I}}$ is $\left(\sigma_{x}\right)^{\beta}$.

For the non-Hermitian topological system with inversion symmetry, we use the total $Z_{2}$ topological invariant $\eta$ to characterize its topological properties

$$
\begin{aligned}
\eta & =\eta_{k=0} \eta_{k=\pi} \\
& =\frac{\operatorname{Tr}\left[\left[\hat{\mathcal{I}} \cdot H_{0}^{\mathrm{SSH}}[k=0]\right]\right.}{\left.\mid \operatorname{Tr}\left[\hat{\mathcal{I}} \cdot H_{0}^{\mathrm{SSH}}[k=0)\right]\right] \mid} \frac{\operatorname{Tr}\left[\hat{\mathcal{I}} \cdot H_{0}^{\mathrm{SSH}}[k=\pi]\right]}{\left.\operatorname{Tr}\left[\hat{\mathcal{I}} \cdot H_{0}^{\mathrm{SSH}}[k=\pi)\right]\right] \mid} \\
& =\frac{t_{1}^{2}-t_{2}^{2}}{\left|t_{1}^{2}-t_{2}^{2}\right|} .
\end{aligned}
$$

There are also two phases, topological phase with $\eta=-1$ in the region of $\left|t_{1}\right|<\left|t_{2}\right|$ and trivial phase with $\eta=1$ in the region of $\left|t_{1}\right|>\left|t_{2}\right|$. At $\left|t_{1}\right|=\left|t_{2}\right|$, there exists a topological phase transition. See the phase diagram in Fig. 1(a).

\section{Effective edge Hamiltonian for defective edge states}

To characterize the two edge states for SSH model under non-Hermitian similarity transformation, we calculate the effective edge Hamiltonian,

$$
\breve{\mathcal{H}}_{\mathrm{eff}}=\left(\begin{array}{cc}
\varepsilon_{L L}^{\prime} & \varepsilon_{L R}^{\prime} \\
\varepsilon_{R L}^{\prime} & \varepsilon_{R R}^{\prime}
\end{array}\right)
$$

where $\varepsilon_{L L}^{\prime}=\left\langle\mathrm{e}_{0}^{\mathrm{L}}\left|H^{\mathrm{SSH}}\right| \mathrm{e}_{0}^{\mathrm{L}}\right\rangle, \varepsilon_{L R}^{\prime}=\left\langle\mathrm{e}_{0}^{\mathrm{L}}\left|H^{\mathrm{SSH}}\right| \mathrm{e}_{0}^{\mathrm{R}}\right\rangle$, $\varepsilon_{R L}^{\prime}=\left\langle\mathrm{e}_{0}^{\mathrm{R}}\left|H^{\mathrm{SSH}}\right| \mathrm{e}_{0}^{\mathrm{L}}\right\rangle, \varepsilon_{R R}^{\prime}=\left\langle\mathrm{e}_{0}^{\mathrm{R}}\left|H^{\mathrm{SSH}}\right| \mathrm{e}_{0}^{\mathrm{R}}\right\rangle$. Here, the basis of the wave-functions of end states are same to those for the Hermitian case [59], i.e., $\left|\mathrm{e}_{0}^{\mathrm{L}}\right\rangle$ and $\left|\mathrm{e}_{0}^{\mathrm{R}}\right\rangle$. For this model, we have

$$
\begin{aligned}
& \varepsilon_{L L}^{\prime}=-\varepsilon_{R R}^{\prime}=0, \\
& \varepsilon_{L R}^{\prime}=e^{\beta} \Delta, \varepsilon_{R L}^{\prime}=e^{-\beta} \Delta .
\end{aligned}
$$

where

$$
\Delta=\frac{\left(t_{2}^{2}-t_{1}^{2}\right)}{t_{2}}\left(\frac{t_{1}}{t_{2}}\right)^{N}
$$

The effective edge Hamiltonian is obtained as

$$
\breve{\mathcal{H}}_{\text {eff }}=\Delta \cdot\left(\tau^{x}\right)^{\beta}
$$

where

$$
\left(\tau^{x}\right)^{\beta}=\cosh (\beta) \tau^{x}+i \sinh (\beta) \tau^{y}=\left(\begin{array}{cc}
0 & \mathrm{e}^{\beta} \\
\mathrm{e}^{-\beta} & 0
\end{array}\right) .
$$



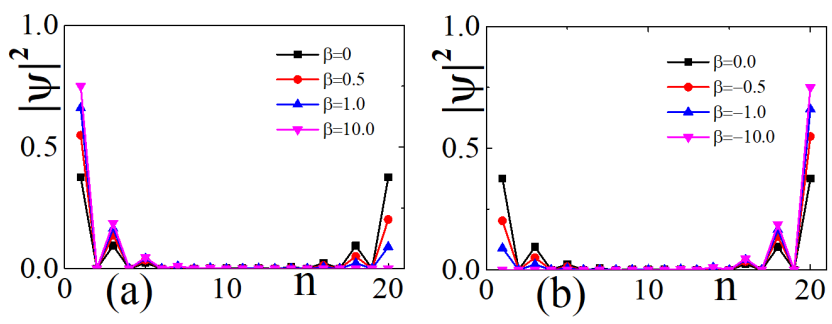

FIG. 2: (Color online) The wave-functions via $\beta$ for edge states of 1D SSH under non-Hermitian similarity transformation: $\beta>0$ for (a) and $\beta<0$ for (b). For this case we have $t_{1}=0.5, t_{2}=1.0$.

Although, the effective edge Hamiltonian $\breve{\mathcal{H}}_{\text {eff }}$ is nonHermitian, i.e., $\breve{\mathcal{H}}_{\text {eff }} \neq\left(\breve{\mathcal{H}}_{\text {eff }}\right)^{\dagger}$, the energy splitting for the edge states doesn't change.

However, the basis of the edge states changes under the non-Hermitian similarity transformation, i.e.,

$$
\begin{aligned}
& \left(\begin{array}{l}
\left|\mathrm{e}_{0}^{\mathrm{L}}\right\rangle \\
\left|\mathrm{e}_{0}^{\mathrm{R}}\right\rangle
\end{array}\right) \rightarrow\left(\begin{array}{l}
\left|\overline{\mathrm{e}}_{0}^{\mathrm{L}}\right\rangle \\
\left.\overline{\mathrm{e}}_{0}^{\mathrm{R}}\right\rangle
\end{array}\right)=S^{-1}\left(\mid \begin{array}{l}
\left|\mathrm{e}_{0}^{\mathrm{L}}\right\rangle \\
\left.\mathrm{e}_{0}^{\mathrm{R}}\right\rangle
\end{array}\right) \\
& =\left(\begin{array}{c}
\left|\mathrm{e}_{0}^{\mathrm{L}}\right\rangle \\
\mathrm{e}^{-\beta}\left|\mathrm{e}_{0}^{\mathrm{R}}\right\rangle
\end{array}\right)
\end{aligned}
$$

where $\left(\begin{array}{c}\left|\overline{\mathrm{e}}_{0}^{\mathrm{L}}\right\rangle \\ \left|\overline{\mathrm{e}}_{0}^{\mathrm{R}}\right\rangle\end{array}\right)$ is the basis for the non-Hermitian case with $\beta \neq 0$. As a result, the eigenstates for the two edge states turn into

$$
\left|\psi_{1}\right\rangle=\frac{1}{\sqrt{1+e^{-2 \beta}}}\left(\left|\mathrm{e}_{0}^{\mathrm{L}}\right\rangle+e^{-\beta}\left|\mathrm{e}_{0}^{\mathrm{R}}\right\rangle\right)
$$

and

$$
\left|\psi_{2}\right\rangle=\frac{1}{\sqrt{1+e^{-2 \beta}}}\left(\left|\mathrm{e}_{0}^{\mathrm{L}}\right\rangle-e^{-\beta}\left|\mathrm{e}_{0}^{\mathrm{R}}\right\rangle\right) .
$$

To characterize the non-Hermitian properties under similarity transformation, the states overlap $\gamma$ between the two edge states is defined as

$$
\gamma=\left\langle\psi_{2} \mid \psi_{1}\right\rangle=\tanh \beta
$$

For the case of $\beta \rightarrow 0$, we have

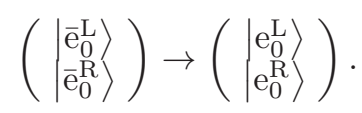

Now, we have $\gamma \rightarrow 0$; On the other hand, for the case of $\beta \rightarrow \infty$, we have

$$
\left(\begin{array}{c}
\left|\overline{\mathrm{e}}_{0}^{\mathrm{L}}\right\rangle \\
\left|\overline{\mathrm{e}}_{0}^{\mathrm{R}}\right\rangle
\end{array}\right) \rightarrow\left(\begin{array}{c}
\left|\mathrm{e}_{0}^{\mathrm{L}}\right\rangle \\
0
\end{array}\right)
$$

Now, we have $\gamma \rightarrow 1$. The edge states become defective: only edge state at left or right end exists.

Fig. 2(a) and Fig. 2(b) show the edge states of the SSH model under non-Hermitian similarity transformation for case of $\beta>0$ and $\beta<0$, respectively. In fact,

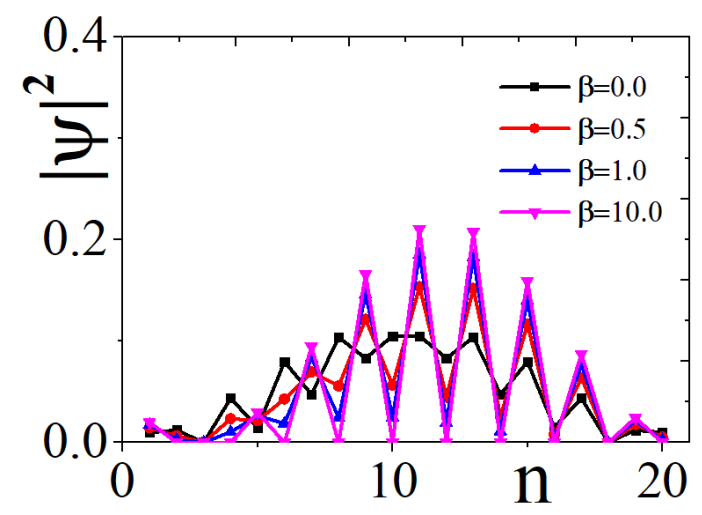

FIG. 3: (Color online) The wave-functions via $\beta$ for bulk states of $1 \mathrm{D}$ SSH under non-Hermitian similarity transformation. For this case, we have $t_{1}=0.5, t_{2}=1.0$.

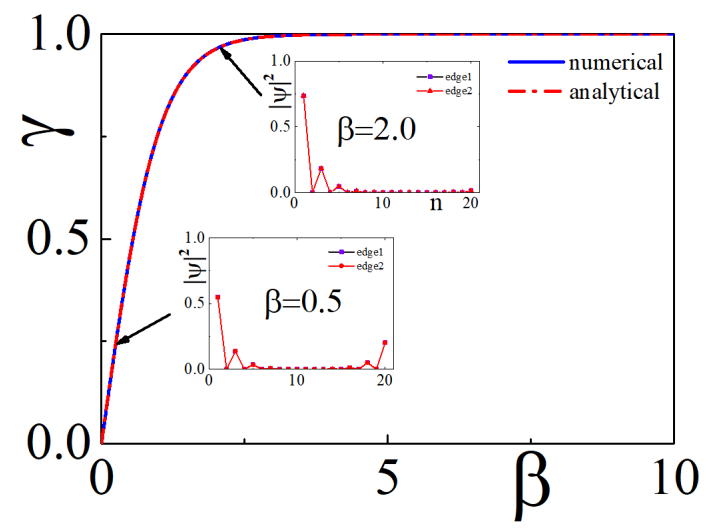

FIG. 4: (Color online) The state overlap $\gamma$ between the two edge states for 1D SSH under non-Hermitian similarity transformation.

the non-Hermitian similarity transformation only polarizes the states onto one selected sublattice in the unit cell. In the strong non-Hermitian limit $\beta \rightarrow \pm \infty$, the edge states localize on the A or B-sublattices that corresponds to a defective edge state on left or right end. Fig. 3 shows the changing of bulk states via $\beta$. From Fig. 3, one can see that there only exists the effect from sublattice polarization, but no non-Hermitian skin effect. The weight of the bulk states tends to accumulate on A-sublattices with increasing $\beta$, and simultaneously decrease on B-sublattice. Fig. 4 shows the states overlap $\gamma$ between the two edge states. From Fig. 4, one can see that the theoretical prediction is consistent to the numerical results. When $\beta \rightarrow 0$, the system reduces to Hermitian SSH model, the states overlap $\gamma$ is zero, while in the strong non-Hermitian limit $\beta \rightarrow \pm \infty$, the states overlap $\gamma$ turns to 1 . This indicates the (singular) defective edge states.

It had been believed that the defective edge states come from the non-Hermitian skin effect. However, for the SSH model under non-Hermitian similarity transformation, without non-Hermitian skin effect in the bulk, 
the defective edge states still exist. Therefore, this result is new.

In addition, we point out that the singular defective edge states with $\gamma=1$ are protected by the chiral symmetry

$$
\sigma_{z} H^{\mathrm{SSH}}(k=0) \sigma_{z}=-H^{\mathrm{SSH}}(k=0)
$$

Due to the chiral symmetry, the wave-function of edge state on left end localizes on A-sublattice and the wavefunction of edge state on right end localizes on Bsublattice. As a result, the non-Hermitian similarity transformation on different sublattices, i.e., $\sigma_{x} \rightarrow$ $\left(\sigma_{x}\right)^{\beta}=\left(\begin{array}{cc}0 & e^{\beta} \\ e^{-\beta} & 0\end{array}\right)$ leads to the non-Hermitian similarity transformation on different edge states, i.e., $\tau_{x} \rightarrow$ $\left(\tau_{x}\right)^{\beta}=\left(\begin{array}{cc}0 & e^{\beta} \\ e^{-\beta} & 0\end{array}\right)$. For this reason, without chiral symmetry, the wave-function of edge state on left/right end no more completely localizes on A/B-sublattice. The non-Hermitian similarity transformation on different sublattices, i.e., $\sigma_{x} \rightarrow\left(\sigma_{x}\right)^{\beta}=\left(\begin{array}{cc}0 & e^{\beta} \\ e^{-\beta} & 0\end{array}\right)$ cannot lead to the non-Hermitian similarity transformation on different edge states, i.e., $\tau_{x} \rightarrow\left(\tau_{x}\right)^{\beta} \neq\left(\begin{array}{cc}0 & e^{\beta} \\ e^{-\beta} & 0\end{array}\right)$. The defective edge states would become normal. Therefore, the singular defective edge states with $\gamma=1$ are unstable against the perturbation breaking the chiral symmetry.

\section{ANOMALOUS BBC AND DEFECTIVE EDGE STATES FOR 2D CHERN INSULATOR UNDER NON-HERMITIAN SIMILARITY TRANSFORMATION}

\section{A. Hermitian deformed Qi-Wu-Zhang model}

Before discussing the defective edge states for 2D nonHermitian Chern insulator, we firstly consider a lattice model (deformed Qi-Wu-Zhang model) of the 2D Hermitian Chern insulator. The Bloch Hamiltonian is given by

$$
\begin{aligned}
H_{0}^{\mathrm{DQWZ}}\left(k_{x}, k_{y}\right) & =\left(v_{x} \sin k_{x}\right) \sigma_{z}+\left(v_{y} \sin k_{y}\right) \sigma_{y} \\
& +\left(\mu-t_{x} \cos k_{x}-t_{y} \cos k_{y}\right) \sigma_{x}
\end{aligned}
$$

where $\sigma_{x, y, z}$ are Pauli matrices. In this paper, we set $t_{x}=t_{y}=v_{x}=v_{y}=1 . \mu$ is the real mass parameter. The eigenvalues of the Hamiltonian can be written as

$E_{ \pm}(\vec{k})= \pm \sqrt{\left(\mu-\cos k_{x}-\cos k_{y}\right)^{2}+\left(\sin k_{x}\right)^{2}+\left(\sin k_{y}\right)^{2}}$

\section{Inversion symmetry and generalized chiral symmetry}

Now, there exists inversion symmetry for $H_{0}^{\mathrm{DQWZ}}\left(k_{x}, k_{y}\right)$ as

$$
\hat{\mathcal{I}} H_{0}^{\mathrm{DQWZ}}\left(k_{x}, k_{y}\right) \hat{\mathcal{I}}^{-1}=H_{0}^{\mathrm{DQWZ}}\left(-k_{x},-k_{y}\right),
$$

where the inversion operator $\hat{\mathcal{I}}$ is $\sigma_{x}$. This equation implies

$$
E(\vec{k})=E(-\vec{k})
$$

In addition, we have a generalized chiral symmetry at $k_{x}=0$, i.e.,

$$
\sigma_{z} H^{\mathrm{DQWZ}}\left(k_{x}=0, k_{y}\right) \sigma_{z}=-H^{\mathrm{DQWZ}}\left(k_{x}=0, k_{y}\right) .
$$

\section{Total $Z_{2}$ topological invariant}

To characterize the $2 \mathrm{D}$ topological insulator, we introduce a new topological invariant - total $Z_{2}$ topological invariant,

$$
\eta=\eta_{\vec{k}=(0,0)} \eta_{\vec{k}=(0, \pi)} \eta_{\vec{k}=(\pi, 0)} \eta_{\vec{k}=(\pi, \pi)} .
$$

See below discussion.

There are four high symmetry points in momentum space, $(0,0),(0, \pi),(\pi, 0),(\pi, \pi)$, at which we have

$$
\hat{\mathcal{I}}|\psi(\vec{k}=(0 / \pi, 0 / \pi))\rangle=|\psi(\vec{k}=(0 / \pi, 0 / \pi))\rangle .
$$

Thus, the Bloch Hamiltonian is divided into two parts

$$
\begin{aligned}
H_{0}^{\mathrm{DQWZ}}(\vec{k}) & =H_{0}^{\mathrm{DQWZ}}(\vec{k} \neq(0 / \pi, 0 / \pi)) \\
+ & H_{0}^{\mathrm{DQWZ}}(\vec{k}=(0 / \pi, 0 / \pi)) .
\end{aligned}
$$

For $\vec{k}=(0,0)$, we have

$$
\frac{1}{2} \operatorname{Tr}\left[\hat{\mathcal{I}} \cdot H_{0}^{\mathrm{DQWZ}}(\vec{k}=(0,0))\right]=\mu-t_{x}-t_{y}=\mu-2
$$

for $\vec{k}=(0, \pi)$, we have

$$
\frac{1}{2} \operatorname{Tr}\left[\hat{\mathcal{I}} \cdot H_{0}^{\mathrm{DQWZ}}(\vec{k}=(0, \pi))\right]=\mu-t_{x}+t_{y}=\mu
$$

for $\vec{k}=(\pi, 0)$, we have

$$
\frac{1}{2} \operatorname{Tr}\left[\hat{\mathcal{I}} \cdot H_{0}^{\mathrm{DQWZ}}(\vec{k}=(\pi, 0))\right]=\mu+t_{x}-t_{y}=\mu
$$

for $\vec{k}=(\pi, \pi)$, we have

$$
\frac{1}{2} \operatorname{Tr}\left[\hat{\mathcal{I}} \cdot H_{0}^{\mathrm{DQWZ}}(\vec{k}=(\pi, \pi))\right]=\mu+t_{x}+t_{y}=\mu+2
$$


To describe the topological structure of the deformed QWZ model $H_{0}^{\mathrm{DQWZ}}(k)$, we define four $Z_{2}$ topological invariants,

$$
\begin{gathered}
\eta_{\vec{k}=(0,0)}=\frac{\operatorname{Tr}\left[\hat{\mathcal{I}} \cdot H_{0}^{\mathrm{DQWZ}}[\vec{k}=(0,0)]\right]}{\left|\operatorname{Tr}\left[\hat{\mathcal{I}} \cdot H_{0}^{\mathrm{DQWZ}}[\vec{k}=(0,0)]\right]\right|}=\frac{\mu-t_{x}-t_{y}}{\left|\mu-t_{x}-t_{y}\right|}, \\
\eta_{\vec{k}=(0, \pi)}=\frac{\operatorname{Tr}\left[\hat{\mathcal{I}} \cdot H_{0}^{\mathrm{DQWZ}}[\vec{k}=(0, \pi)]\right]}{\left|\operatorname{Tr}\left[\hat{\mathcal{I}} \cdot H_{0}^{\mathrm{DQWZ}}[\vec{k}=(0, \pi)]\right]\right|}=\frac{\mu-t_{x}+t_{y}}{\left|\mu-t_{x}+t_{y}\right|}, \\
\eta_{\vec{k}=(\pi, 0)}=\frac{\operatorname{Tr}\left[\hat{\mathcal{I}} \cdot H_{0}^{\mathrm{DQWZ}}[\vec{k}=(\pi, 0)]\right]}{\left|\operatorname{Tr}\left[\hat{\mathcal{I}} \cdot H_{0}^{\mathrm{DQWZ}}[\vec{k}=(\pi, 0)]\right]\right|}=\frac{\mu+t_{x}-t_{y}}{\left|\mu+t_{x}-t_{y}\right|}, \\
\eta_{\vec{k}=(\pi, \pi)}=\frac{\operatorname{Tr}\left[\hat{\mathcal{I}} \cdot H_{0}^{\mathrm{DQWZ}}[\vec{k}=(\pi, \pi)]\right]}{\left|\operatorname{Tr}\left[\hat{\mathcal{I}} \cdot H_{0}^{\mathrm{DQWZ}}[\vec{k}=(\pi, \pi)]\right]\right|}=\frac{\mu+t_{x}+t_{y}}{\left|\mu+t_{x}+t_{y}\right|} .
\end{gathered}
$$

We use the number ' 1 ' to denote the case $\eta_{\vec{k}=(0 / \pi, 0 / \pi)}=$ -1 and the number ' 0 ' to denote the case $\eta_{\vec{k}=(0 / \pi, 0 / \pi)}=$ 1. Hence, there are totally 16 different cases which represent 16 different universal classes of topological states denoted by (1111), (1110), (1101), (1101), (0111), (1100), (1001), (0011), (0110), (0101), (1010), (1000), (0100), (0010), (0001), (0000). The total $Z_{2}$ topological invariant is defined as

$$
\begin{aligned}
\eta & =\eta_{\vec{k}=(0,0)} \eta_{\vec{k}=(0, \pi)} \eta_{\vec{k}=(\pi, 0)} \eta_{\vec{k}=(\pi, \pi)} \\
& =\left\{\begin{array}{l}
+1, \text { trivial phase } \\
-1, \text { topological phase }
\end{array}\right.
\end{aligned}
$$

Then, $\eta$ becomes the topological invariant to characterize the universal properties of 16 different topological orders, of which there are 8 trivial phase : (1111), (1100), (1001), (0011), (0110), (0101), (1010), (0000), and 8 topological phase, (1110), (1101), (1101), (0111), (1000), (0100), (0010), (0001).

For above deformed Qi-Wu-Zhang model with $t_{x}=$ $t_{y}=v_{x}=v_{y}=1$, the total $Z_{2}$ topological invariant is obtained as

$$
\eta=\eta_{\vec{k}=(0,0)} \eta_{\vec{k}=(0, \pi)} \eta_{\vec{k}=(\pi, 0)} \eta_{\vec{k}=(\pi, \pi)}=\frac{\mu^{2}-4}{\left|\mu^{2}-4\right|} .
$$

There are two phases, topological phase with $\eta=-1$ in the region of $\mu^{2}<4$ and trivial phase with $\eta=1$ in the region of $\mu^{2}>4$. At $\mu= \pm 2$, there exists a topological phase transition.

\section{Effective edge Hamiltonian}

In the topological phase $\left(\mu^{2}<4\right)$, we firstly write down the effective Hamiltonian of the edge states.

For open boundary condition along $y$-direction, the topological phase exhibits edge modes localized on the boundaries. For the edge states with wave vector $k_{x}$, we define the basis

$$
\left(\left|\begin{array}{l}
\mathrm{e}_{0, k_{x}}^{\mathrm{L}} \\
\mathrm{e}_{0, k_{x}}^{\mathrm{R}}
\end{array}\right\rangle\right)
$$

The effective Hamiltonian of edge states for Hermitian 2D Chern insulator is

$$
\hat{\mathcal{H}}_{\text {eff }}=\tau^{z} \varepsilon_{k_{x}}+\tau^{x} \Delta_{k_{x}},
$$

where $\varepsilon_{k}= \pm \sin k_{x}$ is the dispersion of the edge states of semi-infinite system and $\Delta_{k_{x}} \sim\left(\mu-\cos k_{x}\right)^{N_{y}}$ is the tunneling strength where $N_{y}$ is the number of lattice sites along $y$-direction. As a result, the energy levels are

$$
\Delta E= \pm \sqrt{\left(\sin k_{x}\right)^{2}+\left(\Delta_{k_{x}}\right)^{2}} .
$$

In thermodynamic limit $N_{y} \rightarrow \infty, \Delta_{k_{x}} \rightarrow 0$, we have

$$
\hat{\mathcal{H}}_{\text {eff }} \rightarrow \tau^{z} \sin k_{x} \text {. }
$$

\section{B. Deformed Qi-Wu-Zhang model under non-Hermitian similarity transformation}

Next, we consider the deformed QWZ model under non-Hermitian similarity transformation $S=\left(\begin{array}{cc}1 & 0 \\ 0 & e^{\beta}\end{array}\right)$ where $\beta$ denotes non-Hermiticity strength.

Under the non-Hermitian similarity transformation, the Hamiltonian of deformed QWZ model turns into

$$
\begin{aligned}
H_{0}^{\mathrm{DQWZ}} & \rightarrow S^{-1} H_{0}^{\mathrm{DQWZ}} S=H^{\mathrm{DQWZ}} \\
& =\left(v_{x} \sin k_{x}\right) \sigma_{z}+\left(v_{y} \sin k_{y}\right)\left(\sigma_{y}\right)^{\beta} \\
& +\left(\mu-t_{x} \cos k_{x}-t_{y} \cos k_{y}\right)\left(\sigma_{x}\right)^{\beta} .
\end{aligned}
$$

The eigenvalues for the Hamiltonian $H^{\mathrm{DQWZ}}$ for the nonHermitian QWZ model are same to those for the Hamiltonian $H_{0}^{\mathrm{DQWZ}}$, i.e.,

$$
E_{ \pm}(k)= \pm \sqrt{\left(\mu-\cos k_{x}-\cos k_{y}\right)^{2}+\left(\sin k_{x}\right)^{2}+\left(\sin k_{y}\right)^{2}} .
$$

Fig. 5 shows the energy spectra of deformed QWZ model under non-Hermitian similarity transformation that are all real and unchanged with $\beta$.

\section{Inversion symmetry and generalized chiral symmetry}

For the non-Hermitian deformed QWZ model, there also exists the inversion symmetry for $H^{\mathrm{DQWZ}}\left(k_{x}, k_{y}\right)$, i.e.,

$$
\hat{\mathcal{I}} H^{\mathrm{DQWZ}}\left(k_{x}, k_{y}\right) \hat{\mathcal{I}}^{-1}=H^{\mathrm{DQWZ}}\left(-k_{x},-k_{y}\right)
$$

where the inversion operator $\hat{\mathcal{I}}$ is $\left(\sigma_{x}\right)^{\beta}$, and a generalized chiral symmetry at $k_{x}=0$, i.e.,

$$
\sigma_{z} H^{\mathrm{DQWZ}}\left(k_{x}=0, k_{y}\right) \sigma_{z}=-H^{\mathrm{DQWZ}}\left(k_{x}=0, k_{y}\right) .
$$




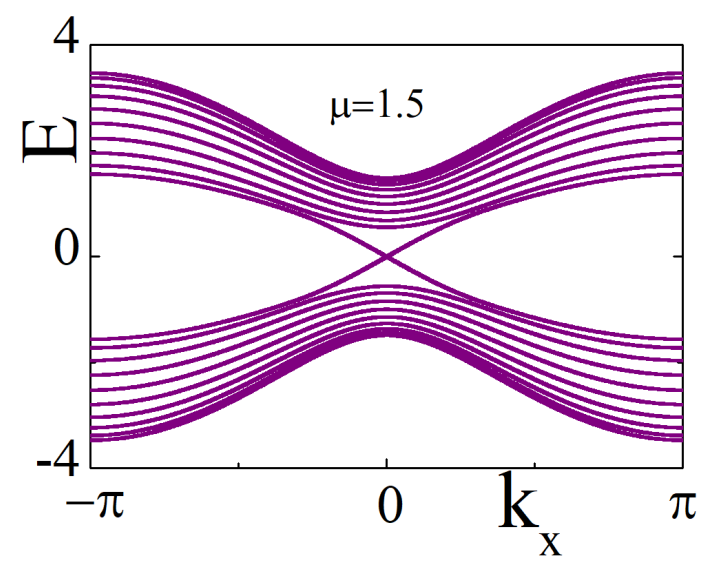

FIG. 5: (Color online) The energy spectra for 1D SSH model under non-Hermitian similarity transformation. The energy spectra are independent on $\beta$.

\section{Total $Z_{2}$ topological invariant}

For non-Hermitian deformed QWZ model with inversion symmetry, we use the total $Z_{2}$ topological invariant $\eta$ to characterize its topological properties,

$$
\begin{aligned}
\eta & =\eta_{\vec{k}=(0,0)} \eta_{\vec{k}=(0, \pi)} \eta_{\vec{k}=(\pi, 0)} \eta_{\vec{k}=(\pi, \pi)} \\
& =\frac{\operatorname{Tr}\left[\hat{\mathcal{I}} \cdot H^{\mathrm{DQWZ}}[\vec{k}=(0,0)]\right]}{\left|\operatorname{Tr}\left[\hat{\mathcal{I}} \cdot H^{\mathrm{DQWZ}}[\vec{k}=(0,0)]\right]\right|} \\
& \cdot \frac{\operatorname{Tr}\left[\hat{\mathcal{I}} \cdot H^{\mathrm{DQWZ}}[\vec{k}=(0, \pi)]\right]}{\left|\operatorname{Tr}\left[\hat{\mathcal{I}} \cdot H^{\mathrm{DQWZ}}[\vec{k}=(0, \pi)]\right]\right|} \\
\cdot & \frac{\operatorname{Tr}\left[\hat{\mathcal{I}} \cdot H^{\mathrm{DQWZ}}[\vec{k}=(\pi, 0)]\right]}{\left|\operatorname{Tr}\left[\hat{\mathcal{I}} \cdot H^{\mathrm{DQWZ}}[\vec{k}=(\pi, 0)]\right]\right|} \\
& \cdot \frac{\operatorname{Tr}\left[\hat{\mathcal{I}} \cdot H^{\mathrm{DQWZ}}[\vec{k}=(\pi, \pi)]\right]}{\left|\operatorname{Tr}\left[\hat{\mathcal{I}} \cdot H^{\mathrm{DQWZ}}[\vec{k}=(\pi, \pi)]\right]\right|} \\
& =\frac{\mu^{2}-4}{\left|\mu^{2}-4\right|}
\end{aligned}
$$

The global phase diagram doesn't change, i.e., topological phase with $\eta=-1$ in the region of $\mu^{2}<4$ and trivial phase with $\eta=1$ in the region of $\mu^{2}>4$. At $\mu= \pm 2$, there exists a topological phase transition.

\section{Effective edge Hamiltonian for defective edge states}

In topological phase $\left(\mu^{2}<4\right)$, the effective Hamiltonian of edge states for the non-Hermitian 2D Chern insulator becomes

$$
\breve{\mathcal{H}}_{\text {eff }}=\tau^{z} \varepsilon_{k_{x}}+\left(\tau^{x}\right)^{\beta} \Delta_{k_{x}},
$$

where

$$
\left(\tau^{x}\right)^{\beta}=\cosh (\beta) \tau^{x}+i \sinh (\beta) \tau^{y}=\left(\begin{array}{cc}
0 & \mathrm{e}^{\beta} \\
\mathrm{e}^{-\beta} & 0
\end{array}\right) .
$$

This effective edge Hamiltonian is also non-Hermitian, i.e.,

$$
\breve{\mathcal{H}}_{\text {eff }} \neq \breve{\mathcal{H}}_{\text {eff }}^{\dagger}
$$

The energy levels for the edge states are

$$
\Delta E= \pm \sqrt{\left(\sin k_{x}\right)^{2}+\left(\Delta_{k_{x}}\right)^{2}}
$$

that are same to the Hermitian case with $\beta=0$. In Fig. 5 , the spectra of edge states are given.

However, the basis for the edge states changes under non-Hermitian similarity transformation, i.e., for the edge states with wave vector $k_{x}$

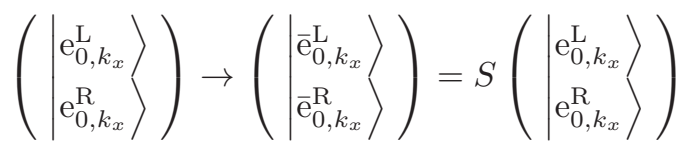

$$
\begin{aligned}
& =\left(\begin{array}{c}
\left|\mathrm{e}_{0, k_{x}}^{\mathrm{L}}\right\rangle \\
\mathrm{e}^{-\beta}\left|\mathrm{e}_{0, k_{x}}^{\mathrm{R}}\right\rangle
\end{array}\right) \text {, }
\end{aligned}
$$

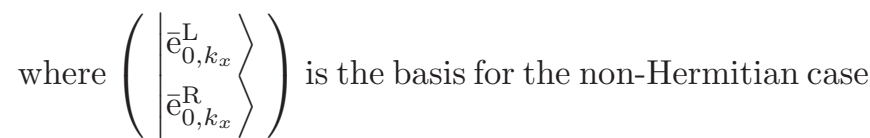
with $\beta \neq 0$. To characterize the non-Hermitian properties from similarity transformation, we also define the states overlap $\gamma_{k_{x}}$ between the two edge states with wave vector $k_{x}$ to be

$$
\gamma_{k_{x}}=\left\langle\psi_{k_{x}, 2} \mid \psi_{k_{x}, 1}\right\rangle=\tanh \beta
$$

For the case of $\beta \rightarrow 0$, we have

$$
\left(\mid \begin{array}{l}
\left|\overline{\mathrm{e}}_{0, k_{x}}^{\mathrm{L}}\right\rangle \\
\left.\overline{\mathrm{e}}_{0, k_{x}}^{\mathrm{R}}\right\rangle
\end{array}\right) \rightarrow\left(\left|\begin{array}{l}
\mathrm{e}_{0, k_{x}}^{\mathrm{L}} \\
\mathrm{e}_{0, k_{x}}^{\mathrm{R}}
\end{array}\right\rangle\right)
$$

Now, we have $\gamma_{k_{x}} \rightarrow 0$; On the other hand, for the case of $\beta \rightarrow \infty$, we have

$$
\left(\mid \begin{array}{l}
\left|\overline{\mathrm{e}}_{0, k_{x}}^{\mathrm{L}}\right\rangle \\
\left.\overline{\mathrm{e}}_{0, k_{x}}^{\mathrm{R}}\right\rangle
\end{array}\right) \rightarrow\left(\begin{array}{c}
\left|\mathrm{e}_{0, k_{x}}^{\mathrm{L}}\right\rangle \\
0
\end{array}\right)
$$

Now, we have $\gamma_{k_{x}} \rightarrow 1$. All edge states with different wave vectors become defective: only edge states at left or right boundary exists.

Fig. 6(a), 6(b) show the edge states along $y$-direction of deformed QZW model under non-Hermitian similarity transformation. One can see that the non-Hermitian similarity transformation will polarize the states spin in degrees of freedom. In the strong non-Hermitian limit $\beta \rightarrow \pm \infty$, the edge states are polarized in spin degrees of freedom that corresponds to a defective edge states 

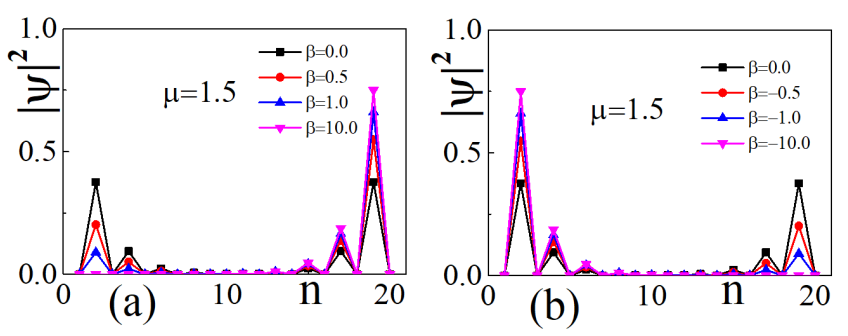

FIG. 6: (Color online) The wave-functions via $\beta$ for edge states of $2 \mathrm{D}$ deformed QWZ model under non-Hermitian similarity transformation: $\beta>0$ for (a) and $\beta<0$ for (b). For this case, we have $t_{x}=t_{y}=v_{x}=v_{y}=1.0$, at $\mu=1.5$.

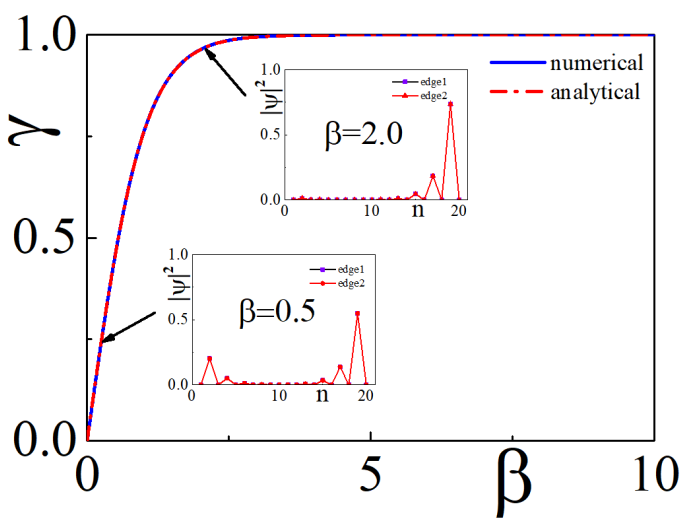

FIG. 7: (Color online) The state overlap $\gamma$ between the two edge states with same wave vector $k_{x}=0.3$ for deformed QZW model under non-Hermitian similarity transformation .

on left/right end. Fig. 7 shows the states overlap $\gamma_{k_{x}}$ between the edge states $\psi_{k_{x}, 1}$ and $\psi_{k_{x}, 2}$ with the same wave vector $k_{x}=0.3$. From Fig. 7 , one can see that the theoretical prediction is also consistent to the numerical results. In the strong non-Hermitian limit $\beta \rightarrow \pm \infty$, $\gamma_{k_{x}}=\left\langle\psi_{k_{x}, 2} \mid \psi_{k_{x}, 1}\right\rangle$ turns to 1 . This indicates the (singular) defective edge states.

For 2D non-Hermitian topological insulators, the intrinsic defective edge states have not been discovered yet. This is an example (in particular, in the strong nonHermitian limit $\beta \rightarrow \pm \infty$ ), of which there only exist edge states on left or right end. In addition, the defective edge states are protected by a generalized chiral symmetry. For the edge states along $y$-direction without a generalized chiral symmetry for the edge states at $k_{y}=0$, i.e.,

$$
\sigma_{z} H^{\mathrm{DQWZ}}\left(k_{x}, k_{y}=0\right) \sigma_{z} \neq-H^{\mathrm{DQWZ}}\left(k_{x}, k_{y}=0\right),
$$

there doesn't exist defective edge states any more.

\section{Usual bulk-boundary correspondence for traditional non-Hermitian Qi-Wu-Zhang model under ST}

In addition, we consider the traditional non-Hermitian Qi-Wu-Zhang model under similarity transformation. The Bloch Hamiltonian is

$$
\begin{aligned}
H^{\mathrm{TQWZ}} & =\left(v_{x} \sin k_{x}\right)\left(\sigma_{x}\right)^{\beta}+\left(v_{y} \sin k_{y}\right)\left(\sigma_{y}\right)^{\beta} \\
& +\left(\mu-t_{x} \cos k_{x}-t_{y} \cos k_{y}\right) \sigma_{z}
\end{aligned}
$$

The energy spectra for the bulk states of this Hamiltonian can be written as

$$
E_{ \pm}(k)= \pm \sqrt{\left(\mu-\cos k_{x}-\cos k_{y}\right)^{2}+\left(\sin k_{x}\right)^{2}+\left(\sin k_{y}\right)^{2}} .
$$

For the non-Hermitian traditional QWZ model, there also exists the inversion symmetry for $H^{\mathrm{DQWZ}}\left(k_{x}, k_{y}\right)$, i.e.,

$$
\hat{\mathcal{I}} H^{\mathrm{DQWZ}}\left(k_{x}, k_{y}\right) \hat{\mathcal{I}}^{-1}=H^{\mathrm{DQWZ}}\left(-k_{x},-k_{y}\right)
$$

where the inversion operator $\hat{\mathcal{I}}$ is $\sigma_{z}$. However, there doesn't exist a generalized chiral symmetry at $\vec{k}=0$, i.e.,

$$
\begin{aligned}
\sigma_{z} H^{\mathrm{TQWZ}}\left(k_{x}=0, k_{y}\right) \sigma_{z} \neq-H^{\mathrm{TQWZ}}\left(k_{x}=0, k_{y}\right), \\
\sigma_{z} H^{\mathrm{TQWZ}}\left(k_{x}, k_{y}=0\right) \sigma_{z} \neq-H^{\mathrm{TQWZ}}\left(k_{x}, k_{y}=0\right) .
\end{aligned}
$$

For traditional Qi-Wu-Zhang model under nonHermitian similarity transformation, the total $Z_{2}$ topological invariant can be also used to characterize its topological properties,

$$
\begin{aligned}
\eta & =\eta_{\vec{k}=(0,0)} \eta_{\vec{k}=(0, \pi)} \eta_{\vec{k}=(\pi, 0)} \eta_{\vec{k}=(\pi, \pi)} \\
& =\frac{\operatorname{Tr}\left[\hat{\mathcal{I}} \cdot H^{\mathrm{TQWZ}}[\vec{k}=(0,0)]\right]}{\left|\operatorname{Tr}\left[\hat{\mathcal{I}} \cdot H_{0}^{\mathrm{TQWZ}}[\vec{k}=(0,0)]\right]\right|} \\
& \cdot \frac{\operatorname{Tr}\left[\hat{\mathcal{I}} \cdot H^{\mathrm{TQWZ}}[\vec{k}=(0, \pi)]\right]}{\left|\operatorname{Tr}\left[\hat{\mathcal{I}} \cdot H^{\mathrm{TQWZ}}[\vec{k}=(0, \pi)]\right]\right|} \\
& \cdot \frac{\operatorname{Tr}\left[\hat{\mathcal{I}} \cdot H^{\mathrm{TQWZ}}[\vec{k}=(\pi, 0)]\right]}{\left|\operatorname{Tr}\left[\hat{\mathcal{I}} \cdot H^{\mathrm{TQWZ}}[\vec{k}=(\pi, 0)]\right]\right|} \\
& \cdot \frac{\operatorname{Tr}\left[\hat{\mathcal{I}} \cdot H^{\mathrm{TQWZ}}[\vec{k}=(\pi, \pi)]\right]}{\left|\operatorname{Tr}\left[\hat{\mathcal{I}} \cdot H^{\mathrm{TQWZ}}[\vec{k}=(\pi, \pi)]\right]\right|} \\
& =\frac{\mu^{2}-4}{\left|\mu^{2}-4\right|} .
\end{aligned}
$$

There are also two phases, topological phase with $\eta=-1$ in the region of $\mu^{2}<4$ and trivial phase with $\eta=1$ in the region of $\mu^{2}>4$. At $\mu= \pm 2$, there exists a topological phase transition.

In topological phase, the effective Hamiltonian of edge states becomes

$$
\breve{\mathcal{H}}_{\mathrm{eff}}=\tau^{z} \varepsilon_{k_{x}}+\tau^{x} \Delta_{k_{x}} .
$$



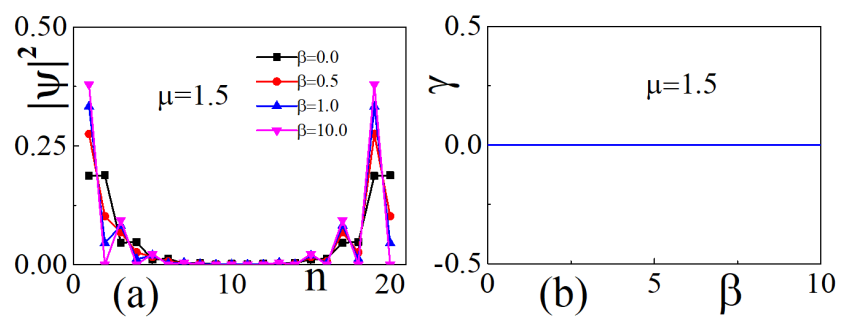

FIG. 8: (Color online) (a) The wave-functions via $\beta$ for edge states of $2 \mathrm{D}$ traditional QWZ model under non-Hermitian similarity transformation. For this case, we have $t_{x}=t_{y}=$ $v_{x}=v_{y}=1.0$, at $\mu=1.5$; (b) The state overlap $\gamma$ between the two edge states.

This effective edge Hamiltonian is Hermitian, i.e.,

$$
\breve{\mathcal{H}}_{\text {eff }}=\breve{\mathcal{H}}_{\text {eff }}^{\dagger}
$$

The energy levels are

$$
\Delta E= \pm \sqrt{\left(\sin k_{x}\right)^{2}+\left(\Delta_{k_{x}}\right)^{2}}
$$

Except for the non-Hermitian spin polarization effect, the basis for the edge states doesn't change under nonHermitian similarity transformation, i.e., for the edge states with wave vector $k_{x}$

$$
\left(\left|\begin{array}{l}
\left.\mathrm{e}_{0, k_{x}}^{\mathrm{L}}\right\rangle \\
\mathrm{e}_{0, k_{x}}^{\mathrm{R}}
\end{array}\right\rangle\right) \rightarrow\left(\left|\begin{array}{l}
\left.\overline{\mathrm{e}}_{0, k_{x}}^{\mathrm{L}}\right\rangle \\
\overline{\mathrm{e}}_{0, k_{x}}^{\mathrm{R}}
\end{array}\right\rangle\right)
$$

Now, the state overlap $\gamma_{k_{x}}$ between the two edge states with wave vector $k_{x}$ is trivial,

$$
\gamma_{k_{x}}=\left\langle\psi_{k_{x}, 2} \mid \psi_{k_{x}, 1}\right\rangle \equiv 0
$$

Then we plot the wave-function for edge states under open boundary condition in Fig. 8(a). We find that the wave-function will never localize on one edge and there is no defective edge state. Instead, the edge states have distribution on both edges with non-Hermitian spin polarization effect. Fig. 8(b) shows the overlap $\gamma=$ $\left\langle\psi_{k_{x}, 2} \mid \psi_{k_{x}, 1}\right\rangle$ between the two edge states with wave vector $k_{x}=0.3$. From Fig. $8(\mathrm{~b})$, one can see that the theoretical prediction is also consistent to the numerical results, i.e., $\left\langle\psi_{k_{x}, 2} \mid \psi_{k_{x}, 1}\right\rangle \equiv 0$. This indicates the usual edge states.

\section{ANOMALOUS BBC AND DEFECTIVE EDGE STATES FOR TOPOLOGICAL INSULATOR ON THE $d$-D CUBIC LATTICE UNDER NON-HERMITIAN SIMILARITY TRANSFORMATION}

In above sections, we have discussed the anomalous $\mathrm{BBC}$ and defective edge states for 1D SSH model and 2D (deformed) QWZ under non-Hermitian similarity transformation. In this section, we will generalize the results to topological insulators in higher dimensions under nonHermitian similarity transformation.

\section{A. Non-Hermitian topological insulators on the $d$-D cubic lattice}

Assuming periodic boundary conditions along all directions, we consider the following Hamiltonian for nonHermitian topological insulators on the $d$-D cubic lattice

$$
\begin{aligned}
H_{\text {cubic }}(\vec{k}) & =\sum_{i \neq j}^{d} \sin k_{i} \Gamma_{i}+\sin k_{j}\left(\Gamma_{j}\right)^{\beta} \\
& +\left(\mu-\sum_{i=1}^{d} \cos k_{i}\right)\left(\Gamma_{d+1}\right)^{\beta}
\end{aligned}
$$

where $\Gamma_{\mu}$ denotes the gamma matrices that satisfy $\left\{\Gamma_{\mu}, \Gamma_{\nu}\right\}=2 \delta_{\mu \nu}$ and $\mu$ is the real mass parameter. Here, we have

$$
\begin{gathered}
\Gamma_{j} \rightarrow\left(\Gamma_{j}\right)^{\beta}=\cosh (\beta) \Gamma_{j}+i \sinh (\beta) \Gamma_{d+1}, \\
\Gamma_{d+1} \rightarrow\left(\Gamma_{d+1}\right)^{\beta}=\cosh (\beta) \Gamma_{d+1}-i \sinh (\beta) \Gamma_{j} .
\end{gathered}
$$

The energy spectra for the bulk states of this Hamiltonian can be written as

$$
E_{ \pm}(\vec{k})= \pm \sqrt{\left(\mu-\sum_{i=1}^{d} \cos k_{i}\right)^{2}+\sum_{i}^{d} \sin ^{2} k_{i}}
$$

\section{B. Inversion symmetry and generalized chiral symmetry}

There exists inversion symmetry for $H_{\text {cubic }}(\vec{k})$ as

$$
\hat{\mathcal{I}} H_{\text {cubic }}(\vec{k}) \hat{\mathcal{I}}^{-1}=H_{\text {cubic }}(-\vec{k}),
$$

where the inversion operator $\hat{\mathcal{I}}$ is $\left(\Gamma_{d+1}\right)^{\beta}$. In addition, we have a generalized chiral symmetry for the edge states at $k_{i \neq j}=0$, i.e.,

$$
\Gamma_{j} H_{\text {cubic }}(\vec{k})\left(k_{i \neq j}=0, k_{j}\right) \Gamma_{j}=-H_{\text {cubic }}(\vec{k})\left(k_{i \neq j}=0, k_{j}\right) .
$$

\section{Total $Z_{2}$ topological invariant}

There are $2^{d}$ high symmetry points in momentum space, $(0, \ldots, 0),(0, \ldots, \pi),(0, \ldots \pi, 0), \ldots$, at which we have

$$
\hat{\mathcal{I}}|\psi(\vec{k}=(0 / \pi, \ldots, 0 / \pi))\rangle=|\psi(\vec{k}=(0 / \pi, \ldots, 0 / \pi))\rangle .
$$

To characterize the non-Hermitian topological insulators on the $d$-D cubic lattice, we introduce a new topological invariant - total $Z_{2}$ topological invariant,

$$
\eta=\prod_{\vec{k}=\mathbf{0}} \eta_{\vec{k}=\mathbf{0}}
$$


where $\mathbf{0}$ means the $2^{d}$ high symmetry points in momentum space $(0, \ldots, 0),(0, \ldots, \pi),(0, \ldots \pi, 0), \ldots$

The Bloch Hamiltonian is divided into two parts

$$
\begin{aligned}
H_{\text {cubic }}(\vec{k}) & =H_{\text {cubic }}(\vec{k} \neq(0 / \pi, \ldots, 0 / \pi)) \\
+H_{\text {cubic }}(\vec{k} & =(0 / \pi, \ldots, 0 / \pi))
\end{aligned}
$$

To describe this topological structure of $H_{\text {cubic }}(\vec{k})$, we define $2^{d} Z_{2}$ topological invariants,

$$
\eta_{\vec{k}=\mathbf{0}}=\frac{\operatorname{Tr}\left[\hat{\mathcal{I}} \cdot H_{\text {cubic }}[\vec{k}=\mathbf{0}]\right]}{\left|\operatorname{Tr}\left[\hat{\mathcal{I}} \cdot H_{\text {cubic }}[\vec{k}=\mathbf{0}]\right]\right|} .
$$

For example, $\vec{k}=(0, \ldots, 0)$, we have

$$
\operatorname{Tr}\left[\hat{\mathcal{I}} \cdot H_{\text {cubic }}(\vec{k}=(0, \ldots, 0))\right]=2(\mu-d) .
$$

Now, we use the number ' 1 ' to denote the case $\eta_{\vec{k}=0}=-1$ and the number ' 0 ' to denote the case $\eta_{\vec{k}=0}=1$. Hence, there are totally $2^{d}$ different cases which represent $2^{2^{d}}$ different universal classes of topological states.

The total $Z_{2}$ topological invariant is defined as

$$
\begin{aligned}
\eta & =\prod_{\vec{k}=\mathbf{0}} \eta_{\vec{k}=\mathbf{0}}=\prod_{\vec{k}=\mathbf{0}} \frac{\operatorname{Tr}\left[\hat{\mathcal{I}} \cdot H_{\text {cubic }}[\vec{k}=\mathbf{0}]\right]}{\left|\operatorname{Tr}\left[\hat{\mathcal{I}} \cdot H_{\text {cubic }}[\vec{k}=\mathbf{0}]\right]\right|} \\
& =\left\{\begin{array}{l}
+1, \text { trivial phase } \\
-1, \text { topological phase }
\end{array}\right.
\end{aligned}
$$

Then, $\eta$ becomes topological invariant to characterize the universal properties of different topological orders, of which there are $2^{2^{d}-1}$ trivial phases for the case of $\mu^{2}>d^{2}$, and $2^{2^{d}-1}$ topological phases, $\mu^{2}<d^{2}$. At $\mu= \pm d$, there exists a topological phase transition.

\section{Effective edge Hamiltonian}

In topological phase $(|\mu|<|d|)$, there exists gapless edge states. Along $x_{j}$-direction, the effective edge Hamiltonian becomes

$$
\breve{\mathcal{H}}_{\mathrm{eff}}=\Gamma_{i} \otimes \tau^{z}\left(\sum_{i \neq j} \sin k_{i}\right)+\Gamma_{i} \otimes\left(\tau^{x}\right)^{\beta} \Delta_{k_{i}}
$$

where $\Delta_{k_{i}} \sim\left(\mu-\sum_{i \neq j} \cos k_{i}\right)^{N_{y}}$, and

$$
\left(\tau^{x}\right)^{\beta}=\cosh (\beta) \tau^{x}+i \sinh (\beta) \tau^{y}=\left(\begin{array}{cc}
0 & \mathrm{e}^{\beta} \\
\mathrm{e}^{-\beta} & 0
\end{array}\right) .
$$

This effective edge Hamiltonian is non-Hermitian, i.e.,

$$
\breve{\mathcal{H}}_{\text {eff }} \neq \breve{\mathcal{H}}_{\text {eff }}^{\dagger}
$$

In thermodynamic limit $N_{j} \rightarrow \infty, \Delta_{k_{i}} \rightarrow 0$, we have

$$
\check{\mathcal{H}}_{\mathrm{eff}} \rightarrow \Gamma_{i} \otimes \tau^{z}\left(\sum_{i \neq j} \sin k_{i}\right) .
$$

As a result, the energy levels for edge states become

$$
\Delta E= \pm \sqrt{\left(\sum_{i \neq j} \sin k_{i}\right)+\left(\Delta_{k_{i}}\right)^{2}}
$$

This indicates that the energy spectra for edge states are independent on $\beta$.

\section{E. Defective edge states}

The basis for the edge states with wave vector $k_{i}$ will be changed under the similarity transformation $S^{-1}$, i.e.,

$$
\begin{aligned}
\left(\left|\begin{array}{l}
\left.\mathrm{e}_{0, k_{i}}^{\mathrm{L}}\right\rangle \\
\left.\mathrm{e}_{0, k_{i}}^{\mathrm{R}}\right\rangle
\end{array}\right\rangle\right. & \rightarrow\left(\begin{array}{l}
\left|\overline{\mathrm{e}}_{0, k_{i}}^{\mathrm{L}}\right\rangle \\
\left.\overline{\mathrm{e}}_{0, k_{i}}^{\mathrm{R}}\right\rangle
\end{array}\right)=S^{-1}\left(\mid \begin{array}{c}
\left.\mathrm{e}_{0, k_{i}}^{\mathrm{L}}\right\rangle \\
\left.\mathrm{e}_{0, k_{i}}^{\mathrm{R}}\right\rangle
\end{array}\right) \\
& =\left(\begin{array}{c}
\left|\mathrm{e}_{0, k_{i}}^{\mathrm{L}}\right\rangle \\
\mathrm{e}^{-\beta}\left|\mathrm{e}_{0, k_{i}}^{\mathrm{R}}\right\rangle
\end{array}\right)
\end{aligned}
$$

where for the edge states with wave vector $k_{i}$, $\left(\left|\begin{array}{l}\mathrm{e}_{0, k_{i}}^{\mathrm{L}} \\ \mathrm{e}_{0, k_{i}}^{\mathrm{R}}\end{array}\right\rangle\right)$ is the basis for the Hermitian case with $\beta=0$ and $\left(\left|\begin{array}{l}\overline{\mathrm{e}}_{0, k_{i}}^{\mathrm{L}} \\ \overline{\mathrm{e}}_{0, k_{i}}^{\mathrm{R}}\end{array}\right\rangle\right)$ is the basis for the non-Hermitian case with $\beta \neq 0$.

To characterize the non-Hermitian properties from similarity transformation, we define the state overlap $\gamma_{k_{i}}$ between the two edge states with wave vector $k_{i}$ to be

$$
\gamma_{k_{i}}=\left\langle\psi_{k_{i}, 2} \mid \psi_{k_{i}, 1}\right\rangle=\tanh \beta .
$$

For the case of $\beta \rightarrow 0$, we have

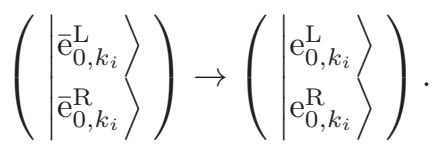

Now, we have $\gamma_{k_{i}} \rightarrow 0$; On the other hand, for the case of $\beta \rightarrow \infty$, we have

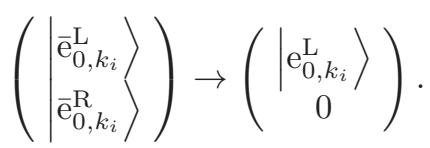

Now, we have $\gamma_{k_{i}} \rightarrow 1$. All edge states become defective: only edge states at left or right boundary.

However, for the edge states along other directions, without generalized chiral symmetry for the edge states at $k_{i}=0$, the edge states become normal.

\section{CONCLUSION}

At the end, we give a brief conclusion. We have exhaustively analyzed a new class of non-Hermitian topological systems: topological insulators under non-Hermitian 
similarity transformation by using 1D SSH model, 2D (Deformed) QWZ model as examples. With the help of a new type of symmetry-protected topological invariant total $Z_{2}$ topological invariant $\eta=\prod_{\vec{k}=\mathbf{0}} \eta_{\vec{k}=\mathbf{0}}\left(\mathbf{0}\right.$ denotes $2^{d}$ high symmetry points in momentum space), the topological phases and trivial phases are classified. In topological phases without non-Hermitian skin effect, there exist defective edge states that are protected by (general) chiral symmetry. The effective edge Hamiltonian $\check{\mathcal{H}}_{\text {eff }}$ are obtained to describe the underlying physics of the defective edge states. The defectiveness of the edge states can be verified by calculating the state overlap $\gamma=\left\langle\psi_{2} \mid \psi_{1}\right\rangle$ between two edge states.
In addition, we point out that the symmetry-protected topological invariant - total $Z_{2}$ topological invariant $\eta$ and the quantitative theory for the defective edge states can be generalized to various types of non-Hermitian topological systems, such as non-Hermitian topological superconductors and non-Hermitian topological semimetals. These issues will be studied in the future.

\section{Acknowledgments}

This work is supported by NSFC Grant No. 11674026 , 11974053.
[1] M. S. Rudner and L. S. Levitov, Phys. Rev. Lett. 102, 065703 (2009).

[2] K. Esaki, M. Sato, K. Hasebe, and M. Kohmoto, Phys. Rev. B 84, 205128 (2011).

[3] M. Sato , K. Hasebe, K. Esaki, and M. Kohmoto, Prog. Theor. Phys. 127, 937 (2012).

[4] Y. C. Hu and T. L. Hughes, Phys. Rev. B 84, 153101 (2011).

[5] S.-D. Liang and G.-Y. Huang, Phys. Rev. A 87, 012118 (2013).

[6] H. Schomerus, Opt. Lett. 38, 1912 (2013) .

[7] B. Zhu, R. Lü, and S. Chen, Phys. Rev. A 89, 062102 (2014).

[8] S. Malzard, C. Poli, and H. Schomerus, Phys. Rev. Lett. 115, 200402 (2015).

[9] T. E. Lee, Phys. Rev. Lett. 116, 133903 (2016).

[10] P. San-Jose, J. Cayao, E. Prada, and R. Aguado, Sci. Rep. 6, 21427 (2016).

[11] A. K. Harter, T. E. Lee, and Y. N. Joglekar, Phys. Rev. A 93, 062101 (2016).

[12] D. Leykam, K. Y. Bliokh, C. Huang, Y. D. Chong, and F. Nori, Phys. Rev. Lett. 118, 040401 (2017).

[13] Y. Xu, S. T. Wang, and L. M. Duan, Phys. Rev. Lett. 118, 045701 (2017).

[14] H. Menke and M. Hirschmann, Phys. Rev. B 95, 174506 (2017) .

[15] H. Shen, B. Zhen, and L. Fu, Phys. Rev. Lett. 120, 146402 (2018).

[16] S. Lieu, Phys. Rev. B 97, 045106 (2018).

[17] Y. Xiong, J. Phys. Commun. 2, 035043 (2018).

[18] K. Kawabata, Y. Ashida, H. Katsura, and M. Ueda, Phys. Rev. B 98, 085116 (2018).

[19] Z. Gong, Y. Ashida, K. Kawabata, K. Takasan, S. Higashikawa, and M. Ueda, Phys. Rev. X 8, 031079 (2018).

[20] S. Yao, and Z. Wang, Phys. Rev. Lett. 121, 086803 (2018).

[21] S. Yao, F. Song, and Z. Wang, Phys. Rev. Lett. 121, 136802 (2018).

[22] F. K. Kunst, E. Edvardsson, J. C. Budich, and E. J. Bergholtz, Phys. Rev. Lett. 121, 026808 (2018).

[23] C. Yin, H. Jiang, L. Li, R. Lü, and S. Chen, Phys. Rev. A 97, 052115 (2018).

[24] K. Kawabata, K. Shiozaki, and M. Ueda, Phys. Rev. B 98, 165148 (2018).
[25] V. M. M. Alvarez, J. E. B. Vargas, M. Berdakin, and L. E. F. F. Torres, Eur. Phys. J. Spec. Top. 227, 1295 (2018).

[26] H. Jiang, C. Yang, and S. Chen, Phys. Rev. A 98, 052116 (2018).

[27] K. Takata and M. Notomi, Phys. Rev. Lett. 121, 213902 (2018).

[28] K. Kawabata, S. Higashikawa, Z. Gong, Y. Ashida, and M. Ueda, Nat. Commun. 10, 297 (2019).

[29] R. Okugawa and T. Yokoyama, Phys. Rev. B 99, 041202(R) (2019).

[30] J. C. Budich, J. Carlström, F. K. Kunst, and E. J. Bergholtz, Phys. Rev. B 99, 041406(R) (2019) .

[31] Z. Yang and J. Hu, Phys. Rev. B 99, 081102(R) (2019) .

[32] A. Ghatak and T. Das, J. Phys.: Condens. Matter 31, 263001 (2019).

[33] J. Avila, F. Peñranda, E. Prada, P. San-Jose, and R. Aguado, Commun. Phys. 2, 1 (2019).

[34] L. Jin and Z. Song, Phys. Rev. B 99, 081103(R) (2019).

[35] S. Lin, L. Jin, and Z. Song, Phys. Rev. B 99, 165148 (2019).

[36] H. C. Wu, L. Jin, and Z. Song, Phys. Rev. B 100, 155117 (2019).

[37] S. Lin, L. Jin, and Z. Song, Phys. Rev. B 99, 165148 (2019).

[38] K. L. Zhang, H. C. Wu, L. Jin, and Z. Song, Phys. Rev. B 100, 045141 (2019).

[39] C. H. Lee and R. Thomale, Phys. Rev. B 99, 201103(R) (2019).

[40] H. Wang, J. Ruan, and H. Zhang, Phys. Rev. B 99, 075130 (2019).

[41] T. Liu, Y. R. Zhang, Q. Ai, Z. Gong, K. Kawabata, M. Ueda, and F. Nori, Phys. Rev. Lett. 122, 076801 (2019).

[42] J. Carlström, M. Stålham mar, J. C. Budich, and E. J. Bergholtz, Phys. Rev. B 99, 161115(R) (2019) .

[43] K. Kawabata, K. Shiozaki, M. Ueda, and M. Sato, Phys. Rev. X 9, 041015 (2019).

[44] H. Zhou and J. Y. Lee, Phys. Rev. B 99, 235112 (2019).

[45] C. H. Liu, H. Jiang, S. Chen, Phys. Rev. B 99, 125103 (2019).

[46] E. Edvardsson, F. K. Kunst, and E. J. Bergholtz, Phys. Rev. B 99, 081302(R) (2019).

[47] L. Herviou, J. H. Bardarson, and N. Regnault, Phys. Rev. A 99, 052118 (2019). 
[48] K. Yokomizo and S. Murakami, Phys. Rev. Lett. 123, 066404 (2019).

[49] F. K. Kunst and V. Dwivedi, Phys. Rev. B 99, 245116 (2019).

[50] S. Longhi, Phys. Rev. Lett. 122, 237601 (2019).

[51] C. H. Lee, L. Li, and J. Gong, Phys. Rev. Lett. 123, 016805 (2019) .

[52] R. Chen, C. Z. Chen, B. Zhou, and D.-H. Xu, Phys. Rev. B 99, 155431 (2019).

[53] T. S. Deng and W. Yi, Phys. Rev. B 100, 035102 (2019).

[54] F. Song, S. Yao, and Z. Wang, Phys. Rev. L 123, 170401 (2019).

[55] X. W. Luo and C. W. Zhang, Phys. Rev. Lett. 123 073601 (2019).

[56] S. Longhi, Phys. Rev. Research 1, 023013 (2019).

[57] M. S. Rudner, M. Levin, and L. S. Levitov, arXiv:1605.0 7652.

[58] Q.-B. Zeng, Y. B. Yang, and Y. Xu, arXiv:1901.08060.

[59] H Jiang, R Lü, S Chen, arXiv:1906.04700.
[60] X. R. Wang, C. X. Guo, and S. P. Kou, arXiv:1912.04024

[61] M. Z. Hasan and C. L. Kane, Rev. Mod. Phys. 82, 3045 (2010).

[62] X. L. Qi and S. C. Zhang, Rev. Mod. Phys. 83, 1057 (2011).

[63] J. Alicea, Rep. Prog. Phys. 75, 076501 (2012).

[64] C. K. Chiu, J. C. Y. Teo, A. P. Schnyder, and S. Ryu, Rev. Mod. Phys. 88, 035005 (2016).

[65] A. Bansil, H. Lin, and T. Das, Rev. Mod. Phys. 88, 021004(2016).

[66] F.M, Fernández, Int J Theor Phys 55, 843 (2016).

[67] W. B. Rui, Y. X. Zhao, and Andreas P. Schnyder, Phys. Rev. B 99, 241110 (2019).

[68] L. F. Liu, B. L. Chen, S. P. Kou. Commun. Theor. Phys. $\mathbf{5 5}, 904(2011)$.

[69] J. Sirker, M. Maiti, N. P. Konstantinidis, and N. Sedlmayr, J. Stat. Mech. P10032 (2014). 